\title{
Fluorescent sensing ochratoxin A with single fluorophore-labeled aptamer
}

\author{
Qiang Zhao $\cdot$ Xia Geng $\cdot$ Hailin Wang
}

Received: 15 March 2013 /Revised: 30 April 2013 / Accepted: 6 May 2013 / Published online: 1 June 2013

(C) Springer-Verlag Berlin Heidelberg 2013

\begin{abstract}
We explored a fluorescent strategy for sensing ochratoxin A (OTA) by using a single fluorophore-labeled aptamer for detection of OTA. This method relied on the change of the fluorescence intensity of the labeled dye induced by the specific binding of the fluorescent aptamer to OTA. Different fluorescein labeling sites of aptamers were screened, including the internal thymine bases, 3'end, and 5'-end of the aptamer, and the effect of the labeling on the aptamer affinity was investigated. Some fluorophorelabeled aptamers showed a signal-on or signal-off response. With the fluorescent aptamer switch, simple, rapid, and selective sensing of OTA at nanomolar concentrations was achieved. OTA spiked in diluted red wine could be detected, showing the feasibility of the fluorescent aptamer for a complex matrix. This method shows potential for designing aptamer sensors for other targets.
\end{abstract}

Keywords Aptamer - Ochratoxin A · Fluorescence · Sensor · Labeling

\section{Introduction}

As novel affinity ligands, aptamers are generated from a nucleic acid library with random sequence, targeting for a wide variety of substances, such as proteins, small molecules,

Electronic supplementary material The online version of this article (doi:10.1007/s00216-013-7047-2) contains supplementary material, which is available to authorized users.

Q. Zhao $(\bowtie) \cdot$ X. Geng

Research Center for Environmental Science and Engineering, Shanxi University, Taiyuan 030006, China

e-mail: chemzhaoq@hotmail.com

H. Wang

State Key Laboratory of Environmental Chemistry and Ecotoxicology, Research Center for Eco-Environmental Sciences, Chinese Academy of Sciences, Beijing 100085, China cells, etc. [1]. The aptamers offer advantages over the commonly used antibodies in easy selection, facile production, good thermal stability, ready and diverse chemical labeling, and low cost. Combining the aptamer affinity and easy signal transduction aptamer-based sensors have attracted wide and increasing interest [2-4]. In the aptamer-based sensors, the socalled signaling aptamers rely on the specific recognition of the targets by the aptamers and the binding-induced signal generations [5-8]. The signal responses of the signaling aptamers are unique properties of the aptamers. The generated signals can be electrochemical, colorimetric, or fluorescent output [3-8]. By using fluorophores on aptamers, the fluorescent signaling aptamers exhibited benefits in sensitivity, simplicity, convenience of detection, diverse detection approaches, and usual homogeneous binding process [5-7, 9-14]. Usually, fluorescent dyes can be readily introduced on the ends of the aptamers through chemical synthesis to fabricate the aptamer switch [5-7, 9-14]. It is also feasible to conjugate fluorophores on the internal bases or to use the fluorescent analogue of the bases in oligonucleotides for sensing $[5-7,15-18]$.

Ochratoxin A (OTA) is a mycotoxin that is found in grains, coffee, wines, beer, etc. The sensitive and rapid detection of OTA is important in food safety, environmental monitoring, and quality control $[19,20]$. Antibody-based methods have been applied to the detection of OTA, such as immunosensors, immunoassays, and immunoaffinity chromatography [19, 20], but the antibody meets limitation in preparation and cost. The recent emergence of the OTA-binding aptamers allows new approaches for OTA detection with great promise [21, 22]. The DNA aptamer for OTA has a high binding affinity, and the dissociation constant reaches about $50 \mathrm{nM}[21,23]$. Many aptamer-based methods for OTA analysis recently have been reported [24-38]. These methods have involved colorimetric, electrochemical, fluorescent, and chromatographic approaches. Among the fluorescent approaches, using fluorophore-labeled aptamers by combination of graphene, carbon nanotubes, organic quenchers, or complementary 
DNA oligos to measure OTA have been demonstrated based on fluorescence quenching or structure switching [37]. In these methods, rational designs and optimal procedures are usually needed.

Using single fluorophore-labeled aptamer to directly detect OTA has not been explored on the basis of the fluorescence change of the fluorescent aptamer resulted from the specific binding of OTA to aptamer. The goal of this work is to use single fluorophore-labeled aptamers for OTA detection by screening the attachment of single dye on different sites of the aptamer against OTA. The labeling sites involved the 3'end, $5^{\prime}$-end, and the internal thymine (T) bases of the aptamer [15]. The responses of the fluorescently labeled aptamers to OTA and the binding affinity of the labeled aptamers were tested. Fluorophore-labeled aptamers with signal-on and signal-off properties were found. OTA at nanomolar could be detected by monitoring the fluorescence change of the fluorescently labeled aptamers. These fluorescent aptamer showed good selectivity and could be applied to the detection of OTA in complex sample matrix, like dilute red wine. The single fluorophore-labeled aptamer switch has advantages in simplicity and rapidity. This study shows potentials for the development of the aptamer sensors for other targets.

\section{Experimental}

\section{Chemical}

OTA, $N$-acety-L-phenylalanine (NAP), and warfarin were obtained from Sigma. The 36-mer DNA aptamer had the following sequence: 5'-GAT CGG GTG TGG GTG GCG TAA AGG GAG CAT CGG ACA-3' [21, 22]. All of DNA aptamers with single 6-carboxyfluorescein (FAM) label were synthesized and purified with HPLC by Sangon Biotech (Shanghai, China). The detailed labeling sites of the aptamer were shown in Table 1 . The ssDNA as control had the following sequence: 5'-CAT CCC CTC TCC CTC CCC TAA ACC CAC CAT CCC GCG-3', and FAM was labeled on $5^{\prime}$-end. Other reagents used in the experiment were analytical grade and supplied by Amresco (Solon, $\mathrm{OH}$ ) or Beijing Chemical Reagents (Beijing, China). Ultrapure water for preparing solution was from a Purelab Ultra Elga Labwater system (VWS Ltd., High Wycombe, Bucks HP14 3JH, UK). The red wine was purchased from local supermarket, and the content of ethanol was $12 \%$.

\section{Fluorometric measurement}

Fluorescence intensity measurement was obtained with F4500 fluorospectrometer (HITACHI, Japan). The experiments were performed at room temperature. For fluorescence intensity measurement, fluorescence emission spectra were recorded with the excitation at $498 \mathrm{~nm}$. The fluorescence intensity at $518 \mathrm{~nm}$ was measured for analysis. The excitation and emission slits were both set at $5 \mathrm{~nm}$. The applied buffer solution contained $10 \mathrm{mM}$ Tris- $\mathrm{HCl}$ (pH 8.5), $120 \mathrm{mM} \mathrm{NaCl}$, $20 \mathrm{mM} \mathrm{CaCl}$, and $0.02 \%$ Tween 20. The dye-labeled aptamer was mixed with varying concentrations of OTA to make the final volume of the solution to be $200 \mu \mathrm{L}$, and then the fluorescence of the dye was recorded by using a $100-\mu \mathrm{L}$ quartz cuvette.

Fluorescence anisotropy (FA) analysis was performed on JASCO FP-8300 fluorescence spectrometer (Japan) by using the L-format configuration, following our previously reported procedure [23]. The experiments were performed at $20{ }^{\circ} \mathrm{C}$. Slits for the excitation and the emission were both set at $5 \mathrm{~nm}$. The FA $(r)$ is defined as $r=\left(I_{\mathrm{VV}}-\mathrm{GI}_{\mathrm{VH}}\right) /\left(I_{\mathrm{VV}}+2 \mathrm{GI}_{\mathrm{VH}}\right)$, where $I$ is the emission intensity, and the subscripts $V$ and $H$ mean the vertical and horizontal orientation of the polarizer, respectively. The first and second subscripts correspond to the orientation of the excitation and the emission polarizers, respectively. The instrumental correction factor $\left(G=I_{\mathrm{HV}} / I_{\mathrm{HH}}\right)$ is applied to the correction of the wavelength response to polarization of the emission optics and detectors. $G$ is dependent on the emission wavelength and spectral bandpass of the emission. FA was measured with an excitation at $375 \mathrm{~nm}$ and an emission at $440 \mathrm{~nm}$ in FA experiments. During FA analysis one $100-\mu \mathrm{L}$ quartz cuvette was employed as the sample cell. Following our early demonstrated approach [23], dissociation constants $\left(K_{\mathrm{d}} \mathrm{s}\right)$ of the fluorescently labeled aptamers were determined through the titration of the OTA with the labeled aptamers in the analysis FA of OTA. The fixed concentration of OTA (62 $\mathrm{nM})$ was mixed with varying concentrations of labeled aptamer in the binding buffer solution (10 mM Tris$\mathrm{HCl}, 120 \mathrm{mM} \mathrm{NaCl}$, and $20 \mathrm{mM} \mathrm{CaCl}_{2}, \mathrm{pH}$ 8.5) with a final volume of $200 \mu \mathrm{L}$. Dissociation constant $\left(K_{\mathrm{d}}\right)$ was finally estimated by nonlinear regression analysis with Graph Pad, shown in Electronic supplementary material (ESM).

\section{Results and discussions}

Principle of detection of OTA using single fluorophore-labeled aptamer

Figure 1 shows the principle of fluorescent sensing OTA with single fluorophore-labeled aptamer. A fluorophore was attached on one site of the aptamer. The specific binding of OTA induces the changes of the conformation of aptamer and subsequent alteration of the environment of the fluorophore, resulting in changes of the fluorescence properties of the fluorophore. Finally, OTA can be measured by monitoring the change of fluorescence signals of the labeled dye. 
Table 1 The sequence of the tested DNA oligos and summary of measured dissociation constants $\left(K_{\mathrm{d}} \mathrm{s}\right)$

\begin{tabular}{lll}
\hline Name & Sequence & $K_{\mathrm{d}}$ \\
\hline O36 & 5'-GAT CGG GTG TGG GTG GCG TAA AGG GAG CAT CGG ACA-3' & $55 \pm 8$ nM [16] \\
5'-FAM-O36 & 5'-FAM-GAT CGG GTG TGG GTG GCG TAA AGG GAG CAT CGG ACA-3' & $125 \pm 11$ nM \\
T3-FAM-O36 & 5'-GAT(FAM) CGG GTG TGG GTG GCG TAA AGG GAG CAT CGG ACA-3' & $1.9 \pm 0.1 \mu M$ \\
T8-FAM-O36 & 5'-GAT CGG GT(FAM)G TGG GTG GCG TAA AGG GAG CAT CGG ACA-3' & $124 \pm 13$ nM \\
T10-FAM-O36 & 5'-GAT CGG GTG T(FAM)GG GTG GCG TAA AGG GAG CAT CGG ACA-3' & $60 \pm 9$ nM \\
T14-FAM-O36 & 5'-GAT CGG GTG TGG GT(FAM)G GCG TAA AGG GAG CAT CGG ACA-3' & $12.2 \pm 0.1 \mu M$ \\
T19-FAM-O36 & 5'-GAT CGG GTG TGG GTG GCG T(FAM)AA AGG GAG CAT CGG ACA-3' & $118 \pm 11$ nM \\
T30-FAM-O36 & 5'-GAT CGG GTG TGG GTG GCG TAA AGG GAG CAT(FAM) CGG ACA-3' & $120 \pm 24$ nM \\
3'-FAM-O36 & 5'-GAT CGG GTG TGG GTG GCG TAA AGG GAG CAT CGG ACA-FAM-3' & $65 \pm 10 \mathrm{nM}$ \\
5'-FAM-S36 & 5'-FAM-CAT CCC CTC TCC CTC CCC TAA ACC CAC CAT CCC GCG-3' & NB \\
\hline
\end{tabular}

$N B$ no binding

The applied 36-mer anti-OTA aptamers had good selectivity and displayed a $K_{\mathrm{d}}$ about $50 \mathrm{nM}$ in the binding buffer solution, as previously reported $[21,23]$. The binding affinity of the aptamer depended on the $\mathrm{Ca}^{2+}$ or $\mathrm{Mg}^{2+}$, and proper ionic strength was also required for the high binding affinity [21, 23]. The previous study showed the optimized binding buffer solution contained $10 \mathrm{mM}$ Tris- $\mathrm{HCl}$ ( $\mathrm{pH}$ 8.5), $20 \mathrm{mM} \mathrm{CaCl}$, and $120 \mathrm{mM} \mathrm{NaCl} \mathrm{[21,} \mathrm{23].}$ Therefore, in this work we applied this optimized binding solution to obtain the high binding affinity of the aptamer to OTA.

Screening the labeling sites

A series of aptamers with single FAM were commercially synthesized. As shown in Table 1, we introduced FAM label at 3'-end, 5'-end, or internal T bases (including T3, T8, T14, T19, and T30) [15]. Figure S1 in the ESM illustrated how the FAM was conjugated on different sites of the aptamer. The present technique allowed the ease of introduction of fluorophores or other functional groups on the internal $\mathrm{T}$ base of DNA oligos by many companies. These singly labeled aptamers included 5'-FAM-O36, T3-FAM-O36, T8-FAMO36, T10-FAM-O36, T14-FAM-O36, T19-FAM-O36, T30-

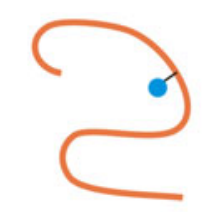

unbound aptamer

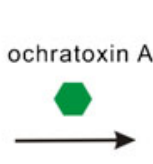

bound aptamer
Fig. 1 Schematic diagram showing the principle for fluorescent sensing OTA with the single fluorophore-labeled aptamer. A fluorophore is attached on one site of the aptamer. The affinity binding of OTA induces the alteration of fluorescence properties of the labeled fluorophore as the consequence of the conformation change of the aptamer
FAM-O36, and 3'-FAM-O36. A FAM-labeled DNA oligo with random sequence (5'-FAM-S36) was also tested as a control.

We investigated the effect of the fluorophore labeling on the binding affinity of the aptamer by using our previously established FA approach taking advantage of the intrinsic fluorescence of OTA [23]. OTA emitted fluorescence at $440 \mathrm{~nm}$ with an excitation at $375 \mathrm{~nm}$ in the binding buffer solution. In this FA approach, OTA at $62 \mathrm{nM}$ was titrated with varying concentrations of aptamers. In the absence of the aptamer, the free OTA showed low FA due to the rapid rotational diffusion. The FA of OTA increased with the addition of aptamers as the result of the increase of molecular volume and restriction of rotation of OTA caused by the binding between OTA and aptamer. The FA increase reflects the binding strength of OTA and aptamer, so FA analysis can tell how the dye labeling on the aptamers affects the binding affinity of aptamer to OTA. Dissociation constants of the aptamers can be determined by the FA titration analysis according to the previously reported strategy [23]. Our previous FA analysis showed the $K_{\mathrm{d}}$ of the unlabeled aptamer was about $55 \mathrm{nM}$ in the tested binding buffer solution [23], consistent with the report from the original selection work [21].

Through the FA titration analysis, we successfully screened the binding affinity of the dye-labeled aptamers. Figure 2 shows the results of the FA titration of OTA by aptamers with FAM labeled on different sites and the control fluorescent DNA oligo (5'-FAM-S36). In the absence of aptamer, the FA value of free OTA was about 0.014, consistent with the previous result [23]. The control DNA did not bind to OTA, so no increase of FA of OTA was observed. The binding affinities of T3-FAM-O36 and T14-FAM-O36 were significantly reduced, as only small FA increase was observed at high concentrations of aptamer. These two $\mathrm{T}$ bases may play key role in maintaining correct conformation of the aptamer or in the interaction between aptamer and OTA. With the addition of the other fluorescently labeled aptamers, the FA of OTA gradually increased, and a plateau 

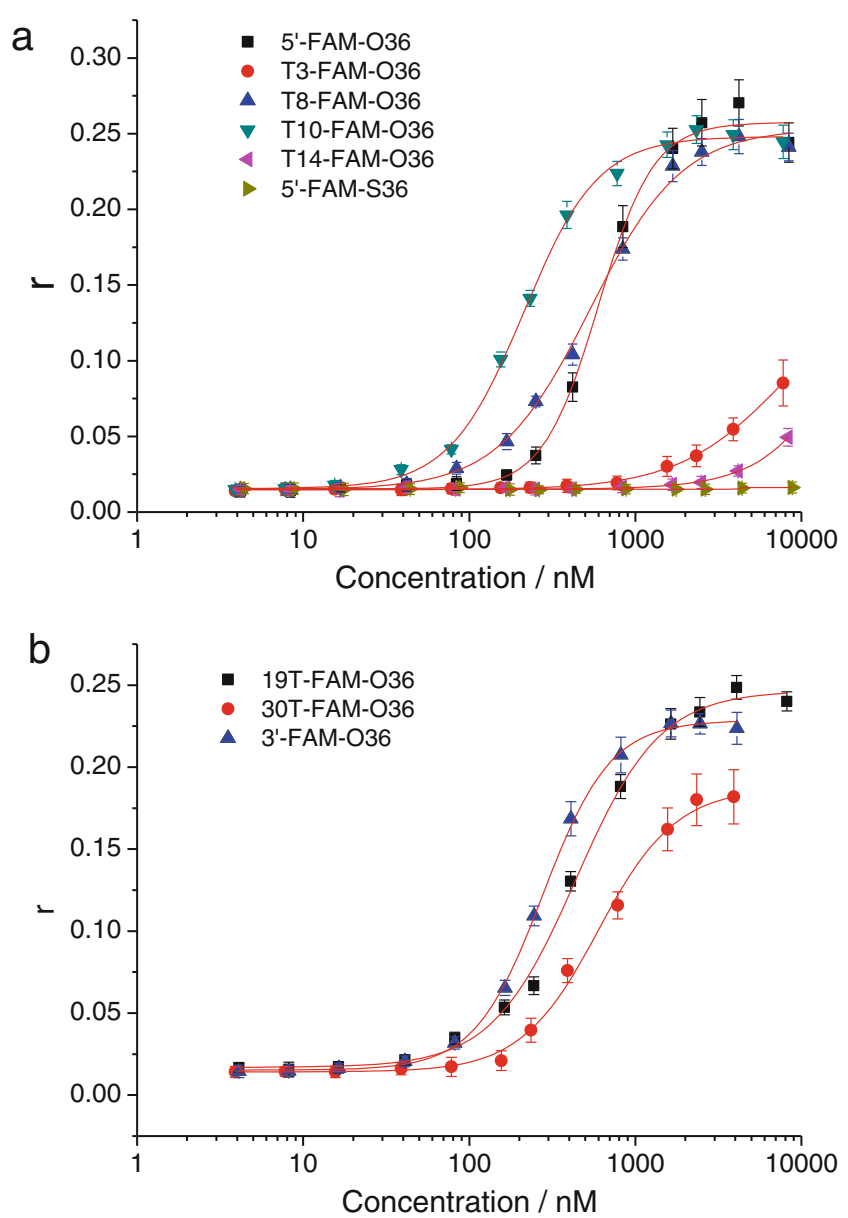

Fig. 2 FA titration of OTA ( $62 \mathrm{nM})$ with increasing concentrations of fluorescently labeled DNA oligos. FA of OTA was measured with emission at $440 \mathrm{~nm}$ and excitation at $375 \mathrm{~nm}$

level was reached at high concentrations of the tested labeled aptamers, corresponding to FA of the totally bound OTA. The obtained plateau level of FA of OTA is larger than the previous result obtained from the unlabeled aptamer [23]. This phenomenon is possibly related to the FRET occurred between OTA and FAM as more quenching of OTA was observed in the presence of FAM-labeled aptamer than the unlabeled aptamer (O36). Fraction of the bound OTA in the solution can be measured through the FA value of the totally bound OTA in the complex with aptamer, FA of OTA in the presence of aptamer, and FA value of totally free OTA (shown in ESM). Through FA titration and the nonlinear regression method (Fig. S2 in the ESM), the $K_{\mathrm{d}} \mathrm{S}$ of the tested aptamers were analyzed and summarized in Table 1 . The obtained $K_{\mathrm{d}} \mathrm{s}$ of $3^{\prime}$-FAM-O36 and T10-FAMO36 were 65 and $60 \mathrm{nM}$, respectively, close to that of the unlabeled 36-mer aptamer (O36), showing no effect of labeling on these locations on the binding affinity of the aptamer for OTA. The $K_{\mathrm{d}} \mathrm{S}$ of T8-FAM-O36, T19-FAMO36, T30-FAM-O36, and 5'-FAM-O36 were about $120 \mathrm{nM}$, suggesting these labeled aptamers still maintain binding affinity to OTA though the binding affinity decreased, compared with the unlabeled aptamers.

The response of fluorescent aptamers to OTA

We tested the response of the fluorescent aptamers to OTA. Upon the addition of OTA, the tested fluorescent aptamers exhibited different behavior. 5'-FAM-O36 and T30-FAMO36 exhibited decrease in fluorescence intensity on the binding to OTA. The fluorescence intensity of T8-FAMO36, T10-FAM-O36, or T19-FAM-O36 increased with the addition of OTA. T3-FAM-O36 and T14-FAM-O36 did not show significant response to OTA as well as the labeled DNA oligo control (5'-FAM-S36), since the above binding affinity analysis shows these fluorescent DNA oligos could not well bind to OTA. In spite of the good binding affinity displayed, the 3'-FAM-O36 exhibited insignificant response
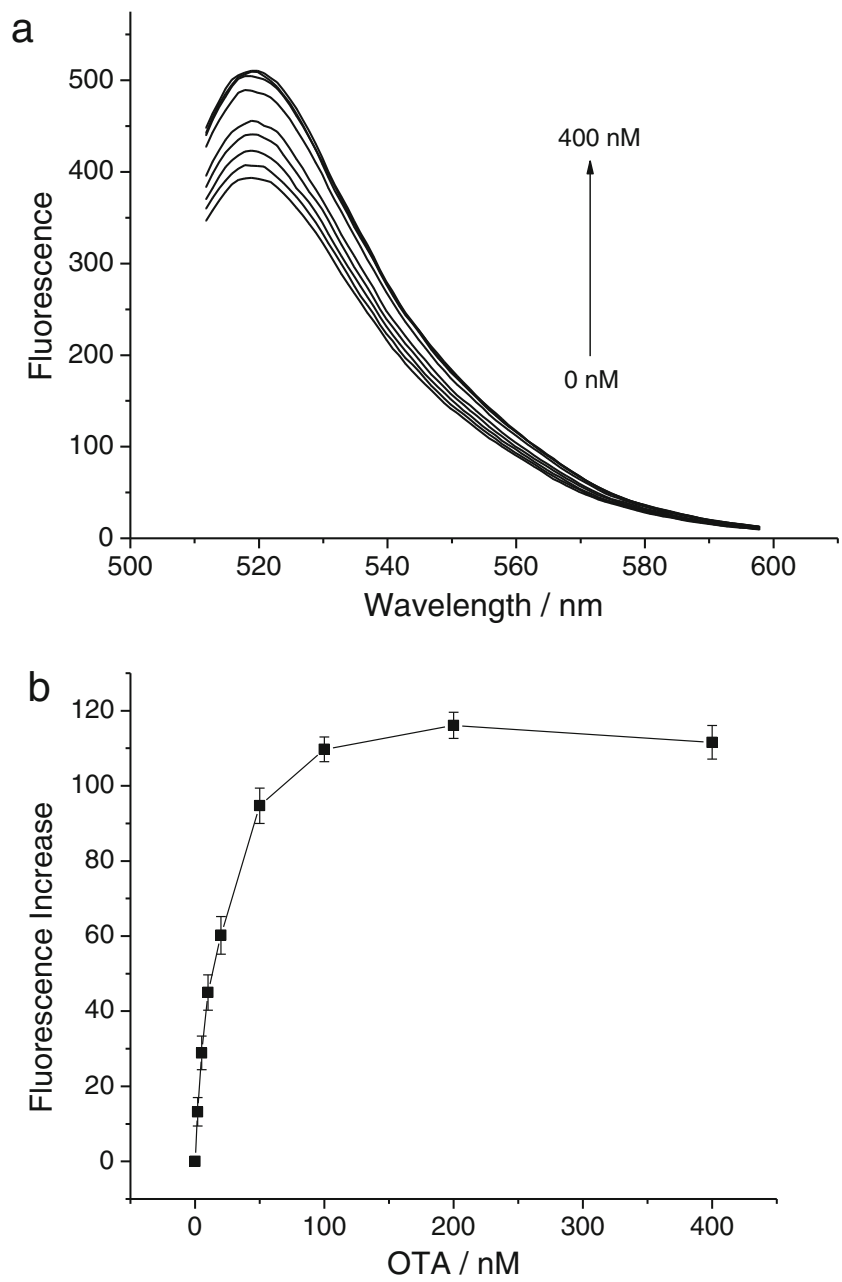

Fig. 3 a Fluorescence spectra of T10-FAM-O36 $(50 \mathrm{nM})$ in the presence of varying concentrations of OTA. From the bottom curve to top curve, the corresponding concentrations of OTA were $0,1,5,10$, $20,50,100,200$, and $400 \mathrm{nM}$. b The fluorescence change of T10FAM-O36 as the function of the concentration of OTA 
to OTA, suggesting no remarked change of the environment of the dye occurred upon the binding of OTA.

Figure 3a shows the responses of T10-FAM-O36 $(50 \mathrm{nM})$ to different concentrations of OTA. With the addition of OTA, the fluorescence intensity of the T10-FAM-O36 increased gradually. The detection range was from 1 to $100 \mathrm{nM}$. Figure $3 \mathrm{~b}$ shows the fluorescence increase as the function of OTA concentration. The observed maximum fluorescence increase was about $28 \%$ of the fluorescence of the aptamer T10FAM-O36. In comparison, T8-FAM-O36 and T19-FAM-O36 allowed detection limits at about $5 \mathrm{nM}$, and the obtained maximum signal increase was $22 \%$ for T19-FAM-O36 or $28 \%$ for T8-FAM-O36 (see Fig. S3 in the ESM). 5'-FAMO36 and T30-FAM-O36 exhibited signal-off response to OTA. The maximum decrease of fluorescence was about $15 \%$ for 5 FAM-O36 or $25 \%$ for T30-FAM-O36 (Fig. 4). For T30-FAMO36, OTA ranging from 2 to $200 \mathrm{nM}$ could be detected (Fig. 4), while OTA from 20 to $200 \mathrm{nM}$ could be detected by
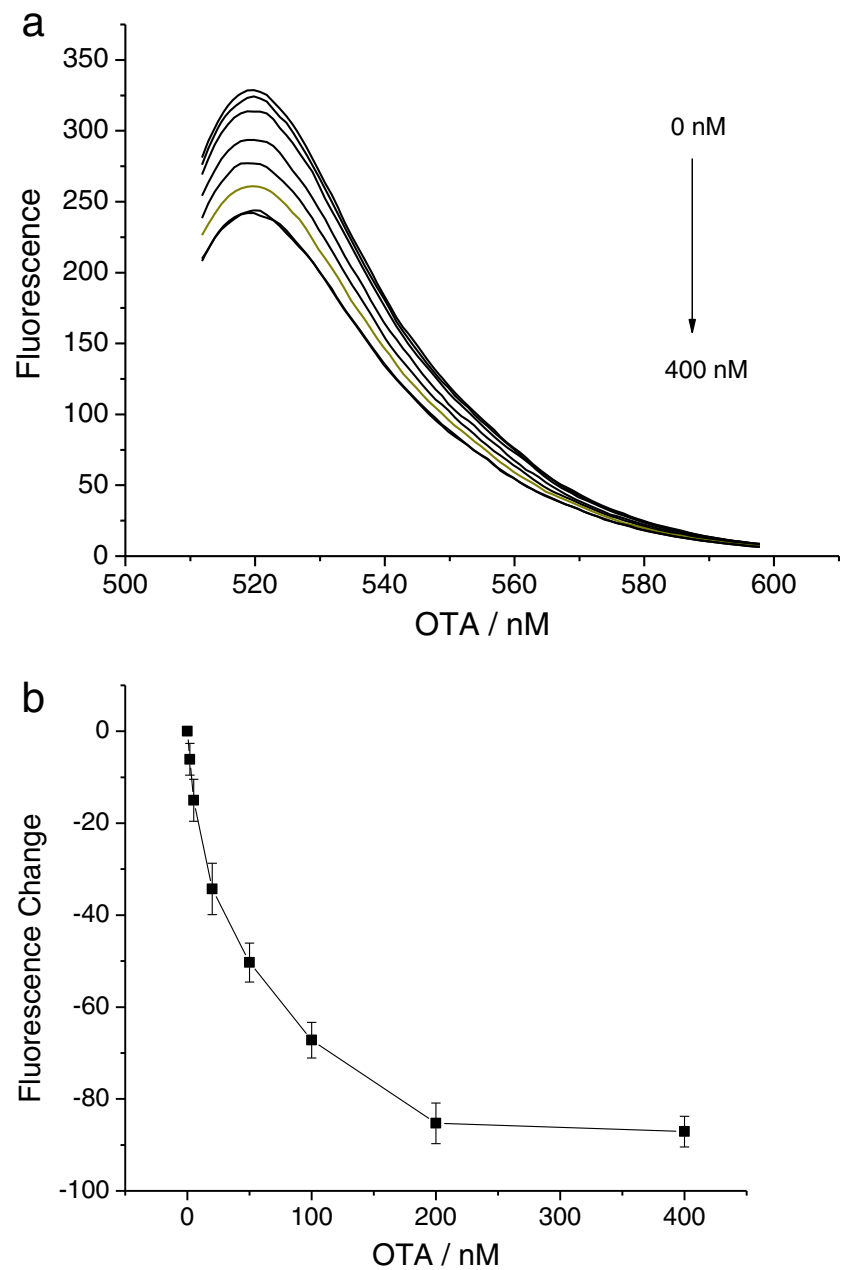

Fig. 4 a Fluorescence spectra of T30-FAM-O36 $(50 \mathrm{nM})$ in the presence of varying concentrations of OTA. From the top curve to bottom curve, the corresponding concentrations of OTA were $0,2,5$, $20,50,100,200$, and $400 \mathrm{nM}$. b The fluorescence change of T30FAM-O36 as the function of the concentration of OTA
5'-FAM-O36 as 5'-FAM-O36 showed less sensitivity. In contrast, upon addition of varying concentrations of OTA, the control 5'-FAM-S36 did not show large change in fluorescence intensity (shown in Fig. S4 in the ESM). Among these aptamers, T10-FAM-O36 gave better signal-on responses, so T10-FAM-O36 was preferred as aptamer probe to sense OTA.

Our approach allows the detection of OTA at nanomolar. Comparing with the other aptamer-based methods for OTA detection [24-38], the sensitivity of our strategy is better than that of the methods using gold nanoparticles (limit of detection (LOD), $22 \mathrm{nM}$ ) [33], graphenes $(1.9 \mu \mathrm{M})$ [29], carbon nanotubes (LOD, $24.5 \mathrm{nM}$ ) [38], and fluorescence ploarization (LOD, $5 \mathrm{nM}$ ) [22], but lower than that of some reported electrochemical methods [30], which allowed the detection of OTA at concentration lower than $1 \mathrm{nM}$. Some research groups recently reported the LOD of OTA reached 0.5 [27] or $0.25 \mathrm{pM}$ [28] by using signal amplification strategy or nanomaterials in the aptamer-based assays. Although the sensitivity was not as high as for methods using signal amplification or nanomaterials, our strategy provides a simple way for sensing OTA at a useful concentration level.

\section{Specificity of fluorescent sensing OTA}

The specificity of OTA sensing with the fluorescent aptamer was examined (Fig. 5). Warfarin and NAP, which had similar structure to OTA, did not cause significant response of the aptamer probe T10-FAM-O36. Amino acids (leucine, aspartate, and histidine) at high concentrations $(0.1 \mathrm{mM})$, and human serum albumin (HSA) at $10 \mu \mathrm{M}$ did not induce the fluorescence increase of the aptamer probe. The results show the response of the fluorescent aptamer is specific for OTA. To further assess whether this method can be applied to the detection of OTA in the complex matrix we made measurement of

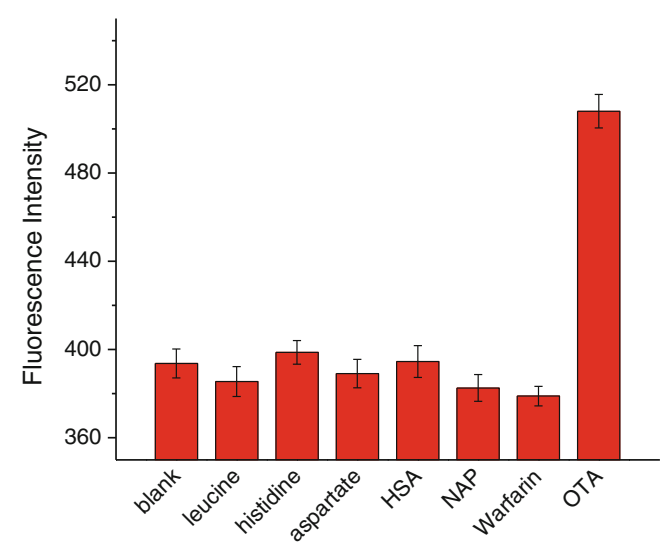

Fig. 5 Specificity test for detection of OTA by using T10-FAM-O36 $(50 \mathrm{nM})$. OTA at $100 \mathrm{nM}$ was analyzed, and amino acids (leucine, histidine, and aspartate) at $0.1 \mathrm{mM}$ and HSA at $0.01 \mathrm{mM}$ were tested. NAP and Warfarin were tested at $175 \mathrm{nM}$ 
OTA $(2,20$, and $100 \mathrm{nM})$ spiked in 100-fold diluted red wine by T10-FAM-O36. The obtained recovery ranged from 90 to $108 \%$, showing the single fluorophorelabeled aptamers may have potentials for OTA analysis in complex matrix. The maximum permitted OTA level for white and red wines is about $2 \mathrm{ng} / \mathrm{mL}(5 \mathrm{nM})$ according to regulation of the European Commission (Commission Regulation (EC) No. 123/2005 of 26 January 2005 amending Regulation (EC) No. 466/2001 as regards OTA) [39]. Our assay needs to improve the sensitivity for real sample analysis. Limitation of the fluorescent aptamers for analysis of OTA still remains as the fluorescent methods usually meet. The presence of components which have fluorescence emission at the same wavelength as labeled fluorophore in the solution could cause interference. Pretreatment of sample may improve the detection performance of the fluorophore-labeled aptamers. For example, selective preconcentration of OTA previous to analysis will help for the analysis of OTA in complex samples.

\section{Conclusions}

In summary, we reported a simple fluorescent strategy for OTA sensing with single-fluorophore-labeled aptamers, relying on the binding-induced fluorescence changes of the aptamer. The aptamer with dye labeled at various sites displayed different response to OTA binding. The effect of the labeling on the binding affinity of the aptamers was also examined. Single fluorophore-labeled aptamer with good binding affinity and response capability was used to detect OTA, allowing the detection of OTA at nanomolar. The dyelabeled aptamer showed good selectivity and could be used in the analysis of OTA in diluted red wine. This work shows potential for detection of other targets by similar strategy.

Acknowledgments This work was supported by grants from the National Natural Science Foundation of China (grant No. 21222503), the State Key Laboratory of Environmental Chemistry and Ecotoxicology in the Research Center for Eco-Environmental Sciences of the Chinese Academy of Sciences (grant No. KF2010-24), and the Key Project of Chinese Ministry of Education (grant No. 212020).

\section{References}

1. Klussmann S (ed) (2006) The aptamer handbook, functional oligonucleotides and their applications. Wiley, Weinheim

2. Li Y, Lu Y (2009) Functional nucleic acids for analytical applications. Springer, New York

3. Liu J, Cao Z, Lu Y (2009) Chem Rev 109:1948-1998

4. Cho EJ, Lee JW, Ellington AD (2009) Annu Rev Anal Chem 2:241-264
5. Jhaveri SD, Kirby R, Conrad R, Maglott EJ, Bowser M, Kennedy RT, Glick G, Ellington AD (2000) J Am Chem Soc 122:2469-2473

6. Nutiu R, Li Y (2004) Chem Eur J 10:1868-1876

7. Juskowiak B (2011) Anal Bioanal Chem 399:3157-3176

8. Lubin AA, Plaxco KW (2010) Acc Chem Res 43:496-505

9. Gokulrangan G, Unruh JR, Holub DF (2005) Anal Chem 77:19631970

10. Heyduk E, Heyduk T (2005) Anal Chem 77:1147-1156

11. Zhu Z, Yang R, You M, Zhang X, Wu Y, Tan W (2010) Anal Bioanal Chem 396:73-83

12. Ruta J, Perrier S, Ravelet C, Fize J, Peyrin E (2009) Anal Chem 81:7468-7473

13. Chang H, Tang L, Wang Y, Jiang J, Li J (2010) Anal Chem 82:2341-2346

14. Wang W, Chen C, Qian M, Zhao XS (2008) Anal Biochem 373:213-219

15. Zhang D, Lu M, Wang H (2011) J Am Chem Soc 133:9188-9191

16. Katilius E, Katiliene Z, Woodbury NW (2006) Anal Chem 78:6484-6489

17. Patel M, Dutta A, Huang H (2011) Anal Bioanal Chem 400:30353040

18. Zhu Z, Ravelet C, Perrier S, Guieu V, Fiore E, Peyrin E (2012) Anal Chem 84:7203-7211

19. Monaci L, Palmisano F (2004) Anal Bioanal Chem 378:96-103

20. Hayat A, Paniel N, Rhouati A, Marty JL, Barthelmebs L (2012) Food Control 26:401-415

21. Cruz-Aguado JA, Penner G (2008) J Agric Food Chem 56:1045610461

22. Cruz-Aguado JA, Penner G (2008) Anal Chem 80:8853-8855

23. Geng X, Zhang D, Wang H, Zhao Q (2013) Anal Bioanal Chem 405:2443-2449

24. Kuang H, Chen W, Xu D, Xu L, Zhu Y, Liu L, Chu H, Peng C, Xu C, Zhu S (2010) Biosens Bioelectron 76:710-716

25. Wang L, Ma W, Chen W, Liu L, Ma W, Zhu Y, Xu L, Kuang H, Xu C (2011) Biosens Bioelectron 26:3059-3062

26. Bone L, Vidal JC, Duato P, Castillo JR (2011) Biosens Bioelectron 26:3254-3259

27. Barthelmebs L, Jonca J, Hayat A, Prieto-Simon B, Marty J-L (2011) Food Control 22:737-743

28. Wang L, Chen W, Ma W, Liu L, Ma W, Zhao Y, Zhu Y, Xu L, Kuang H, Xu C (2011) Chem Commun 47:1574-1576

29. Sheng L, Ren J, Miao Y, Wang J, Wang E (2011) Biosens Bioelectron 26:3494-3499

30. Girolamo AD, McKeague M, Miller JD, DeRosa MC, Visconti A (2011) Food Chem 127:1378-1384

31. Rhouati A, Paniel N, Meraihi Z, Marty JL (2011) Food Control 22:1790-1796

32. Chapuis-Hugon F, du Boisbaudry A, Madru B, Pichon V (2011) Anal Bioanal Chem 400:1199-1207

33. Yang C, Wang Y, Marty JL, Yang XR (2011) Biosen Bioelectron 26:2724-2727

34. Tong P, Zhao WW, Zhang L, Xu JJ, Chen HY (2012) Biosens Bioelectron 33:146-151

35. Wu S, Duan N, Ma X, Xia Y, Wang H, Wang Z, Zhang Q (2012) Anal Chem 84:6263-6270

36. De Girolamo A, Le L, Penner G, Schena R, Visconti A (2012) Anal Bioanal Chem 403:2627-2634

37. Yang XH, Kong WJ, Yang MH, Zhao M, Ouyang Z (2013) Chin J Anal Chem 41:297-306

38. Guo ZJ, Ren JT, Wang JH, Wang EK (2011) Talanta 85:2517_ 2521

39. Tessini C, Mardones C, von Baer D, Vega M, Herlitz E, Saelzer R, Silva J, Torres O (2010) Anal Chim Acta 660:119-126 